\title{
On the origin of the radial flow in low energy heavy ion reactions
}

\author{
Regina Nebauer*+ and Jörg Aichelin* \\ * SUBATECH \\ Laboratoire de Physique Subatomique et des Technologies Associées \\ UMR Universitè de Nantes, IN2P3/CNRS, Ecole des Mines de Nantes \\ 4, rue Alfred Kastler F-44070 Nantes Cedex 03, France. \\ + Universität Rostock, Rostock, Germany
}

\begin{abstract}
The average transverse energy of nucleons and intermediate mass fragments observed in the heavy ion reaction $\mathrm{Xe}(50 \mathrm{~A} \mathrm{MeV})+\mathrm{Sn}$ shows the same linear increase as a function of their mass as observed in heavy ion collisions up to the highest energies available today and fits well into the systematics. At higher energies this observation has been interpreted as a sign of a strong radial flow in an otherwise thermalized system. Investigating the reaction with Quantum Molecular dynamics simulations we find in between $50 \mathrm{~A} \mathrm{MeV}$ and $200 \mathrm{~A} \mathrm{MeV}$ a change in the reaction mechanism. At $50 \mathrm{~A} \mathrm{MeV}$ the apparent radial flow is merely caused by an in-plane flow and Coulomb repulsion. The average transverse fragment energy does not change in the course of the reaction and is equal to the initial fragment energy due to the Fermi motion. At $200 \mathrm{~A} \mathrm{MeV}$, there are two kinds of fragments: those formed from spectator matter and those from the center of the reaction. There the transverse energy is caused by the pressure from the compressed nuclear matter. In both cases we observe a binary event structure, even in central collisions. This demonstrates as well the non thermal character of the reaction. The actual process which leads to multifragmentation is rather complex and is discussed in detail.
\end{abstract}

\section{INTRODUCTION}

It is known since long that for almost all particles observed in heavy ion reactions between $30 \mathrm{~A} \mathrm{MeV}$ and $200 \mathrm{~A} . \mathrm{GeV}$ the transverse kinetic energy spectra have a Maxwell-Boltzmann form, predicted for an emission from an equilibrated source. However, the apparent temperature of the spectra and hence the average kinetic energy of the particles is quite different for different hadrons and fragments and increases with increasing mass and increasing energy. This observation seemed to exclude an identification of the apparent temperature with the real temperature of the system.

Recently it has been conjectured [1] 3] that at all energies between $50 \mathrm{~A} \mathrm{MeV}$ and $200 \mathrm{~A} \mathrm{GeV}$ the assumption of a strong radial flow can reconcile the mass dependence of the apparent temperature with thermodynamics. At relativistic and ultra-relativistic energies this has been inferred by comparing transverse pion, kaon and proton spectra [1]. At energies below $500 \mathrm{~A} \mathrm{MeV}$ the lever arm is still larger because one can include the intermediate mass fragments (IMF's) of masses in between 2 and 10 [2,3], emitted at mid rapidity, to separate radial flow and temperature. The deviations in forward and backward direction are usually interpreted as preequilibrium emission.

This observation has renewed the interest in the thermal analysis of heavy ion reactions in the high as well as in the low energy heavy ion community [2,4]. It revealed a very large value for the radial flow velocity (up to $40 \%$ of the speed of light) and a very peculiar beam energy 
dependence [4] which has been discussed as a possible sign for a transition from a hadronic phase to a Quark Gluon Plasma phase.

Especially at low beam energies the observed value of the radial velocity in units of the speed of light of $.05 \leq \beta_{r} / c \leq 0.2$ [2] (the exact value depends on the event selection) is hard to understand in terms of physical processes because none of the other observables present evidence that the system is sufficiently compressed for attributing the radial flow to an equation of state effect. The very moderate in-plane flow is negative (as in deep inelastic collisions) and points therefore to an other origin than compression and subsequent release of the compressional energy, in contradistinction to higher energies where the in-plane flow is positive. Nevertheless, the low energy points follow smoothly the above mentioned systematics.

This radial flow at 50A MeV has been reproduced [5] by simulations using the Quantum Molecular Dynamics (QMD) [6] approach. The same is true at higher energies where QMD simulations have been performed by the FOPI collaboration [3].

It is the purpose of this article to take advantage of this agreement and to study the origin of the large increase of the transverse energy with the mass and hence the origin of the radial flow. Here we concentrate ourselves on the reaction at $50 \mathrm{~A} \mathrm{MeV}$ and study in detail how the fragments are produced. The reaction mechanism at this low energy is quite different than that at higher energies. To demonstrate this we present as well the same study for a beam energy of 200A MeV. A detailed study of the radial flow at higher energies, its equation of state dependence and its physical origin will be the subject of a forthcoming publication [7].

For our study at $(50 \mathrm{~A} \mathrm{MeV})$ we use simulations of the reaction $X e+S n$ which has recently been measured by the INDRA collaboration. The INDRA detector at GANIL has been constructed to study multifragmentation and therefore the angular coverage and the energy thresholds have been chosen to be better than that of any other $4 \pi$ detector elsewhere. Hence the data taken with this detector are most suitable to confirm or disprove the theories embedded in the simulation programs. A detailed comparison of our results with the experimental data will be published elsewhere [5]. Here we mention that not only the mass dependence of the average kinetic energy but also the kinetic energy spectra themselves are in reasonable agreement with experiment.

For details about the QMD approach we refer to reference [6]. In this program the nucleons are represented by Gaussian wave packets with a constant width. The time evolution of the centers of these wave packets is given by Euler Lagrange equations derived from the Lagrangian of the system. The nucleons have an effective charge of $\mathrm{Z} / \mathrm{A}$. The fragment distribution becomes stable after $240 \mathrm{fm} / \mathrm{c} \mathrm{[8]}$. From this point on we employ a Coulomb trajectory program for the fragments and nucleons until the Coulomb energy is released. This second step is necessary at this low energy because a large fraction of the kinetic energy of the fragments is due to the Coulomb energy. 


\section{THE RADIAL FLOW AT BEAM ENERGIES OF 50A MEV}

A first general idea of the time evolution of the collision can be obtained from the time evolution of the density of the system. If the maximal density is reached the nuclei have their maximal overlap, after that the system expands and the density decreases. This permits to find the time scale of the reaction.

The total density is the sum over all nucleons which are described by Gaussians:

$$
\rho(\vec{r}, t) \propto \sum_{i=1}^{A} e^{-\frac{\left(\vec{r}-\overrightarrow{r_{i}}(t)\right)^{2}}{2 L}}
$$

The width of the Gaussians is $4 L=4.33 \mathrm{fm}^{2}$ and $\mathrm{A}$ is the number of nucleons present in the system.

In figure 1, left hand side, we plot the time evolution of the total density in the center of the reaction. The maximum density is obtained at $\approx 50 \mathrm{fm} / \mathrm{c}$, on the same time scale the system expands and reaches at $120 \mathrm{fm} / \mathrm{c}$ a low density phase where the fragments do not interact anymore.
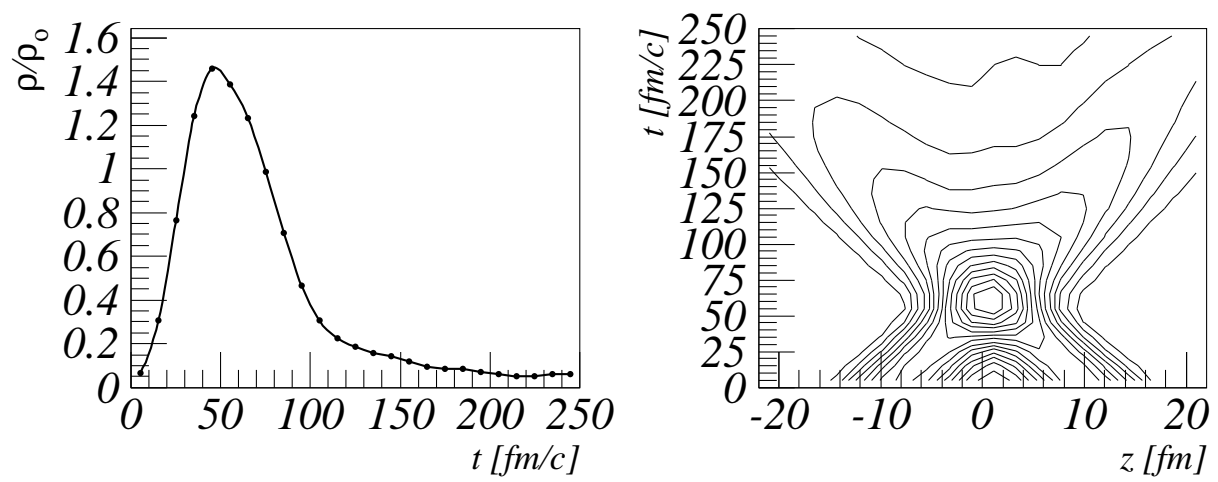

FIG. 1. Time evolution of the density (left) and of the density profile along the beam (z) axis (right) for the system $\mathrm{Xe}(50 \mathrm{~A} \mathrm{MeV})+\mathrm{Sn}, b=3 \mathrm{fm}$.

On the right hand side of the same figure we display the density profile along the beam (z) axis. Here we can follow the two nuclei. They occupy the same coordinate space at $50 . .60 \mathrm{fm} / \mathrm{c}$. The system expands after $120 \mathrm{fm} / \mathrm{c}$. We find that this quasi-central $(b=3 \mathrm{fm})$ collision is semi transparent. Projectile and target pass through each other without being seriously decelerated. For $b=0 \mathrm{fm}$ we get the same result. That binary character is confirmed by experiment: In the center of mass system the experiment shows a flat angular distribution $\left(d N / d \cos \theta_{c m}\right)$ between $60^{\circ} \leq \theta_{c m} \leq 120^{\circ}$ as well as a constant average kinetic energy for fragments $Z \geq 3$ even in central collisions. In forward and backward direction a strongly enhanced cross section is observed. The INDRA collaboration made use of this observation and presented their data in two angular bins: $60^{\circ} \leq \theta_{C M} \leq 120^{\circ}$ (IMF's emitted in this angular range are called mid-rapidity fragments (MRF's)) and $\theta_{C M}<60^{\circ}, \theta_{C M}>120^{\circ}$ called projectile/target like fragments (PTF's) [2].

How do the transverse fragment momenta reflect this passage through the other nucleus? This is displayed in figure 2 for $\mathrm{a} b=3 \mathrm{fm}$ reaction. Here we show the time evolution of the average transverse momentum of all fragments with $5 \leq A \leq 10$ (left) and $A>10$ (right). For 
the dotted points the transverse momentum is represented with respect to the beam direction, for the triangles with respect to the largest eigenvector of the momentum tensor which is tilted by the flow angle $\theta_{\text {flow }}$.

\section{- $p_{t}$ fragments}

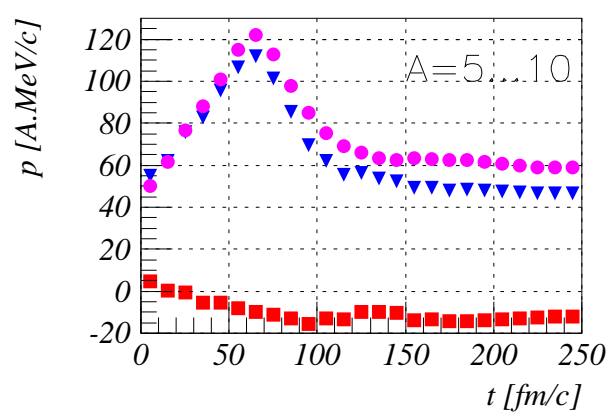

$p_{x} \operatorname{sign}\left(p_{z}\right)$ fragments

$\nabla p_{t}$ rotated system fragments

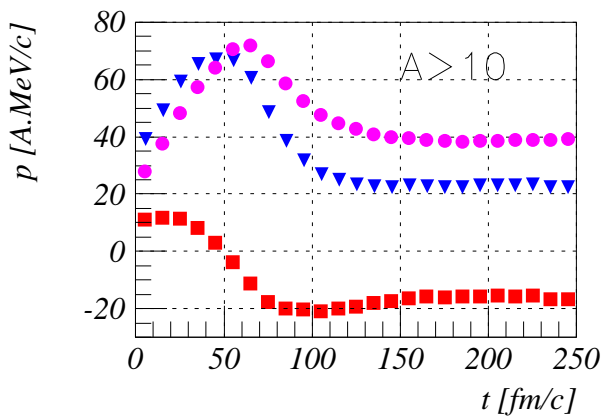

FIG. 2. Time evolution of the average transverse momentum of the IMF's with respect to the beam direction (dots) and in the rotated system (by $\theta_{\text {flow }}$ ) (triangles) for $50 \mathrm{~A} \mathrm{MeVXe}+S n, b=3 \mathrm{fm}$. The squares mark the time evolution of the in-plane flow.

It is the first seminal result of this article that in the rotated system the average transverse momentum is initially and finally the same. A very similar result we obtain for $b=1$ and $b$ $=5 \mathrm{fm}$. During the reaction the particles are accelerated in transverse direction but later they feel a force into the opposite direction. The origin of this acceleration will be discussed later. As squares we display the time evolution of the in-plane flow $\frac{1}{N_{F}} \sum_{N_{F}} \operatorname{sign}\left(p_{z}^{c m}\right) p_{x} . N_{F}$ is the number of fragments. We see that it remains moderate and is negative.

The average transverse energy of the fragments as a function of the fragment charge is displayed in fig. 3. On the left hand side we display the transverse energy with respect to the beam axis, on the right hand side with respect to the rotated system. The mean value is presented after the initialization, after $250 \mathrm{fm} / \mathrm{c}$, when the fragment distribution gets stable, and finally after the mutual Coulomb repulsion has ceased.
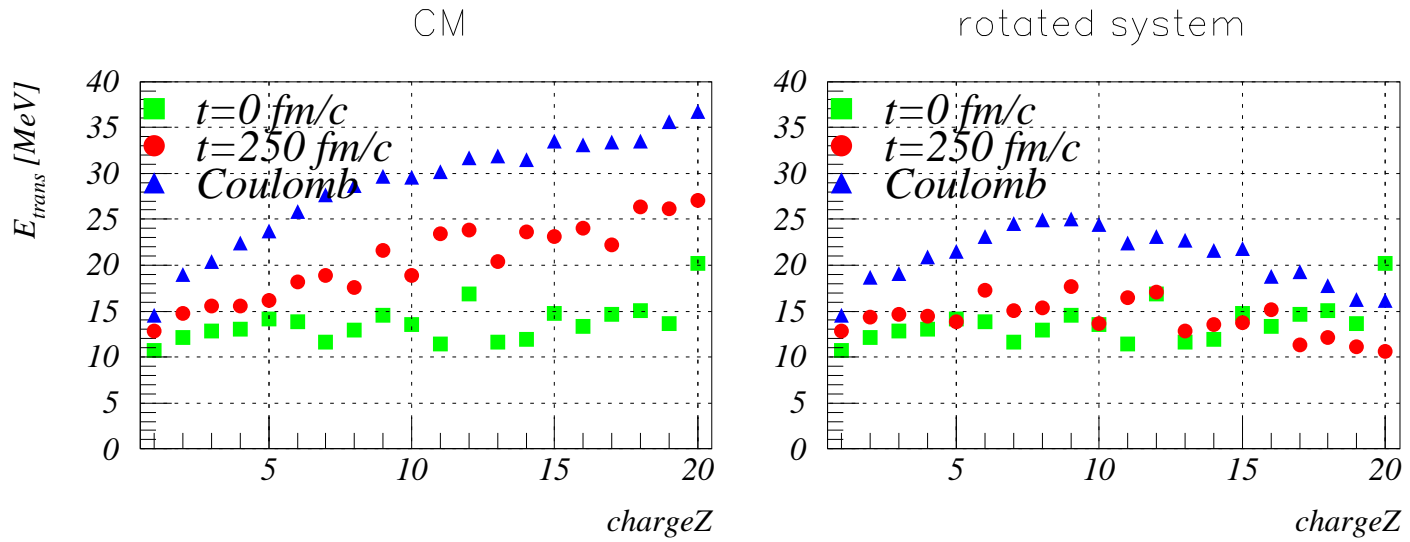

FIG. 3. Average transverse energy of the IMF's as a function of their charge with respect to the beam direction (left) and in the rotated system (by $\theta_{\text {flow }}$ ) (right) for $50 \mathrm{AMeVXe}+S n, b=3 \mathrm{fm}$.

It is the second seminal result of this article that the average transverse fragment energy initially is about $14 \mathrm{MeV}$ and independent of the fragment size. As Goldhaber [9] has pointed 
out many years ago this is expected if the fragment formation is a fast process. In this case the momentum distribution of the fragments is a convolution of the momentum distribution of the entrained nucleons and one expects for the fragment momentum squared

$$
P^{2}=<\left(\sum_{i}^{A} p_{i}\right)^{2}>=\frac{3 k_{\text {Fermi }}^{2}}{5} A \frac{N-A}{N-1}
$$

where $\mathrm{A}$ is the number of nucleons entrained in the fragment and $\mathrm{N}$ is the number of nucleons of the disintegrating nucleus. Hence the fragment kinetic energy is

$$
E_{A}=\frac{3 E_{F e r m i}}{5} \frac{N-A}{N-1}
$$

and as well about $14 \mathrm{MeV}$ in the QMD calculations. (Due to surface effects it differs from the value of a Fermi gas in a square wall potential).

How it can happen that the fragments pass the reaction zone without being heated up and are finally observed at midrapidity or in PTF's we will investigate in the second part of this article. The nucleons interact via the potential

$$
V=-124 \frac{\rho}{\rho_{0}}+70.5\left(\frac{\rho}{\rho_{0}}\right)^{2}[\mathrm{MeV}]
$$

where the density is given by equation 1 and $\rho_{0}$ is the normal nuclear matter density. In order to reveal the physics which drives the reaction we display the relative density of those nucleons which are finally entrained in MRF's or PTF's as a function of time in the x-z plane

$$
\rho_{\text {rel }}^{M R F / P T F}(x, z, t)=\frac{\rho_{M R F / P T F}(x, z, t)}{\rho_{\text {total }}(x, z, t)}
$$

and superimpose the gradient of the potential in the $\mathrm{x}-\mathrm{z}$ plane as arrows where $\mathrm{x}$ is the direction of the impact parameter. For the sake of a clearer display we plot nucleons coming from the projectile only. We start out with a discussion of the reaction at zero impact parameter then we continue with $b=3 \mathrm{fm}$ reactions.

The motion of the nucleons in the potential of a nucleus is a sequence of acceleration and deceleration. Nucleons on the surface are almost at rest, due to the density (and thus the potential) gradient they become accelerated towards the center of the nucleus. They reach their maximal momentum when they pass the center of the nucleus, climb up the potential on the other side and are finally at rest again when arriving at the surface. When a heavy ion collision occurs, the position of the nucleons in the projectile or target determines whether they "feel" the heavy ion collision right from the beginning or only when the high density phase has already passed. We will show that the initial position of the entrained nucleons decides as well whether the fragment is finally observed at mid rapidity or in forward/ backward direction.

In figure 4 we display the motion of the nucleons finally entrained in PTF's for a reaction at $b=0 \mathrm{fm}$. The spatial distribution of those nucleons is almost identical with that of all nucleons present in the projectile. In the first step of the collision the nucleons move away from the target into the yet unperturbed part of the projectile. When they arrive at the back end of the projectile they invert the direction of their momenta. They are then accelerated in longitudinal direction towards the center of the reaction. When they arrive finally there the high density zone has disappeared already. Hence the nucleons pass the center without a larger change of their initial momentum. The initial correlations [10] among the nucleons which finally form a fragment survive the reaction because all potential gradients are small. 


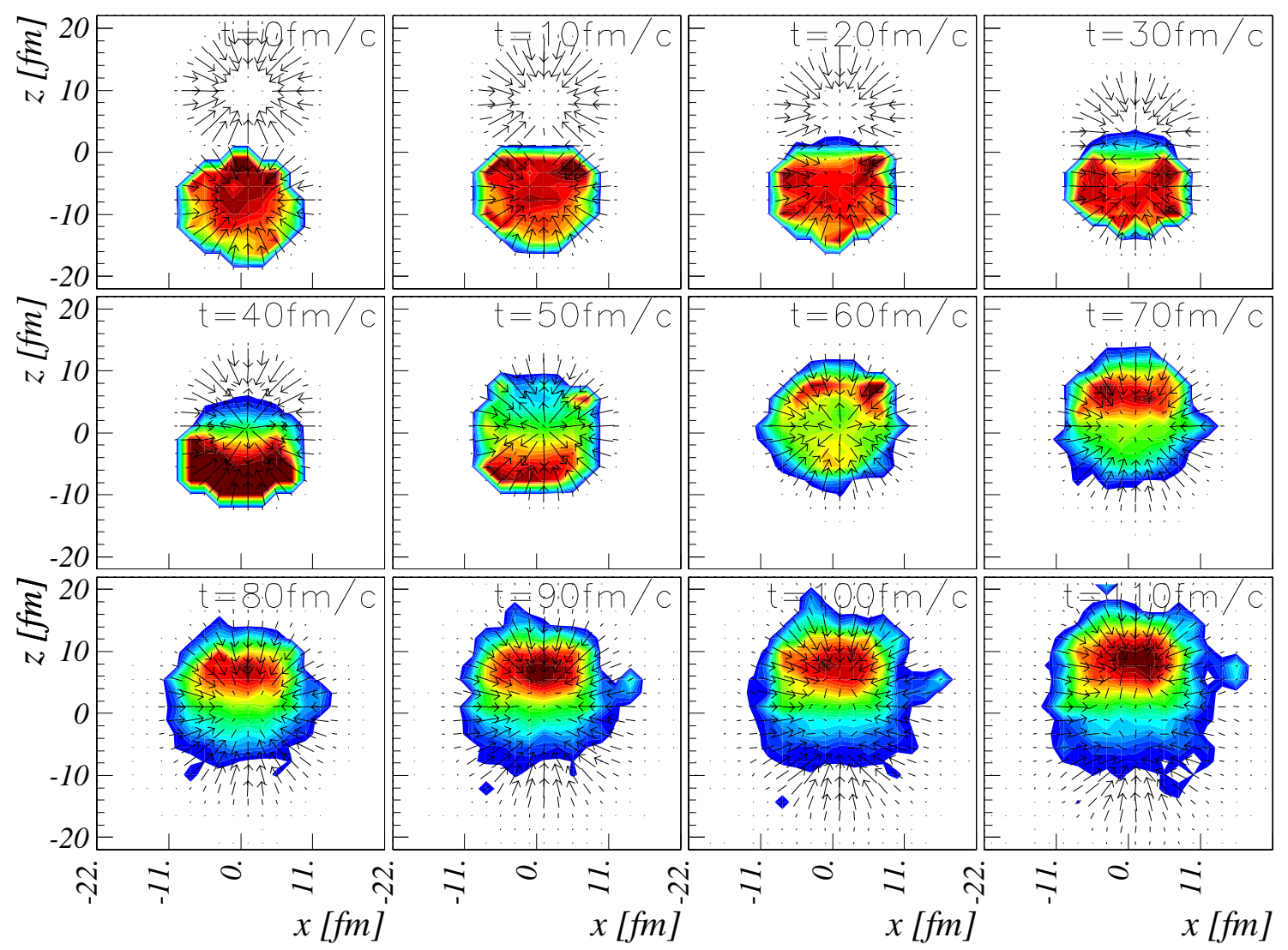

FIG. 4. Reaction $X e(50 \mathrm{~A} \mathrm{MeV})+S n$. Movement of the nucleons finally emitted as IMF's in forward/backward direction in the mean field potential for collisions at impact parameter $b=0 \mathrm{fm}$. We display the fraction of these nucleons on the total density (shadow) and the gradient of the potential (arrows) projected on the $x$-z plane

Nucleons finally emitted as MRF's (fig. 5) are strongly located at the front end of the nuclei. These are the nucleons which climb the nuclear potential created by the higher density in the reaction zone and which are at rest before they are on the top of the potential wall. Due to their position they are involved in the collisions between projectile and target nucleons right from the beginning. Collisions support the deceleration. (Later collisions are to a large extend Pauli suppressed.) They escape the barrier in transverse direction. As their (longitudinal as well as transverse)momentum is quite small, the nucleons stay longer in the center of the reaction what favours the mixing of projectile and target nucleons. When leaving the reaction zone the fragments become decelerated due to the potential interaction with the rest of the system. This deceleration balances the gain in energy due to the prior acceleration in transverse direction, although the physics of both processes is rather independent.

The same analysis for PTF's for $b=3 \mathrm{fm}$ is displayed in figure 6. The general reaction mechanisms, even the time scales, are the same as discussed before. In addition to the scenario at $b=0 \mathrm{fm}$ asymmetry effects occur here. Up to $40 \mathrm{fm} / \mathrm{c}$ the scenario is the same as at zero impact parameter. When the high density phase occurs, the nucleons take the line of least resistance, i.e. they follow the minimum of the potential on the right hand side (for the projectile nucleons, the target nucleons take the inverse direction on the other side). The 


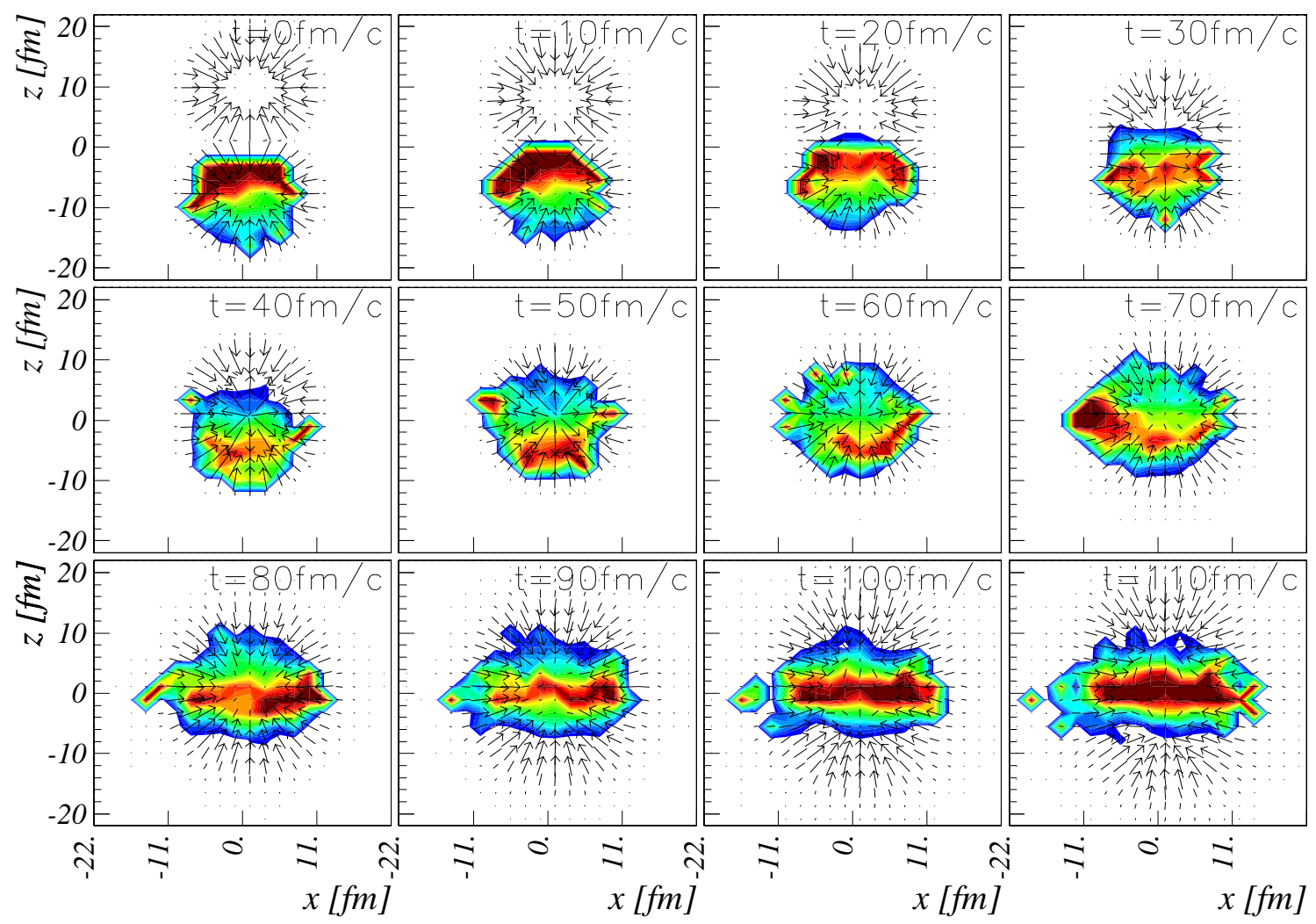

FIG. 5. Reaction Xe(50A MeV)+Sn. Movement of the nucleons finally emitted as IMF's in mid-rapidity in the mean field potential for collisions at impact parameter $b=0 \mathrm{fm}$. We display the fraction of these nucleons on the total density (shadow) and the gradient of the potential (arrows) projected on the $x$-z plane

larger part of the nucleons pass the reaction center when the potential barrier has disappeared. As already discussed for zero impact parameter case, the nucleons emitted as fragments in forward/backward direction pass through the reaction zone without an important change of their initial momenta.

For the reaction $\mathrm{Xe}(50 \mathrm{~A} \mathrm{MeV})+\mathrm{Sn}$ we could find two modes for the emission of fragments:

1. The Fragments are already "preformed" in the nuclei. The initial correlations between the nucleons survive as they avoid the high density zone. For a finite impact parameter a strong in-plane flow is observed. The fragments are quite big and emitted in forward/backward direction.

2. Fragments emitted in midrapidity are smaller. They are formed by mixing projectile and target nucleons. Due to their initially small longitudinal momentum they stay in the high density zone and emitted in transverse direction. These fragments are rather small as they are formed via collisions. 


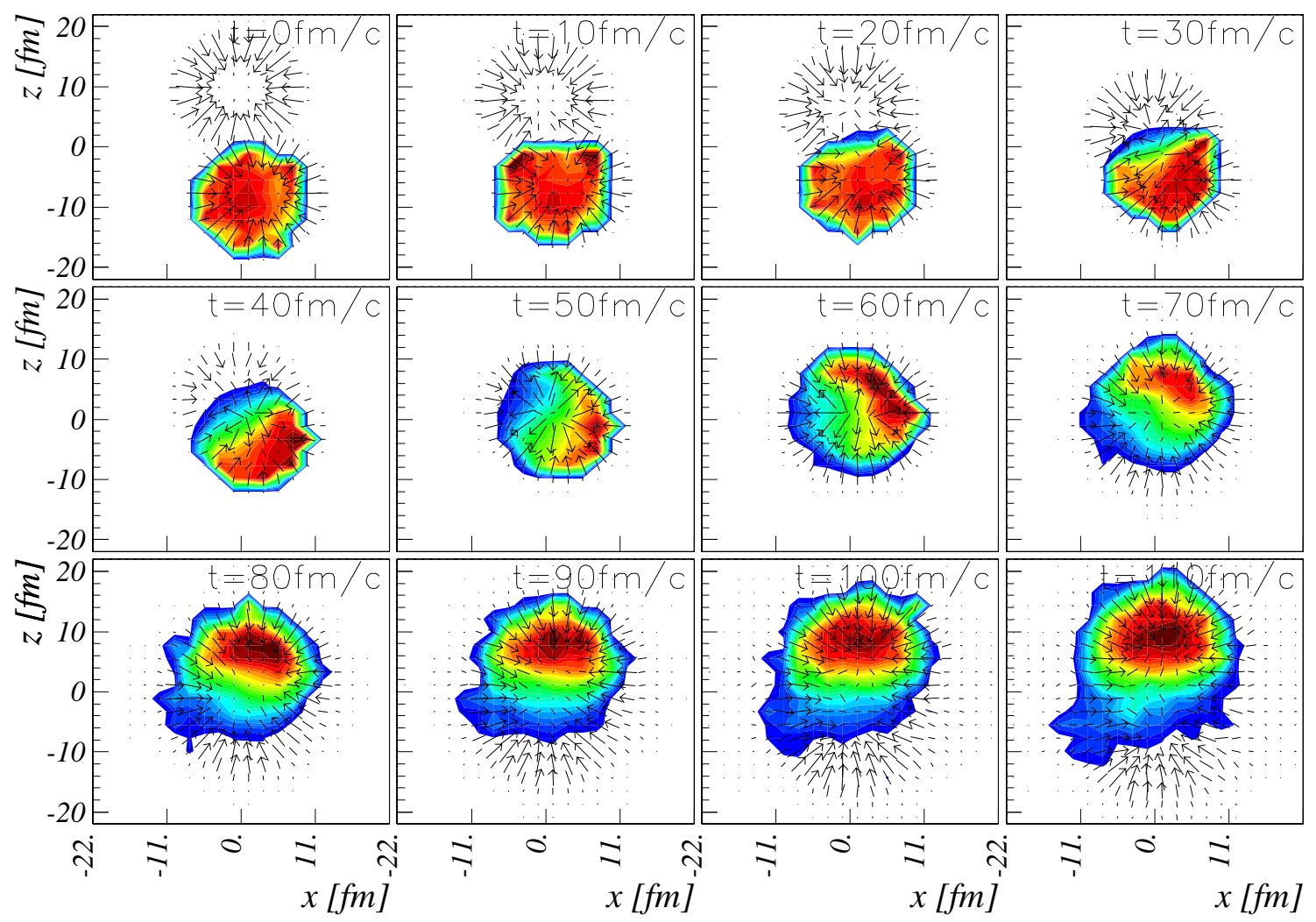

FIG. 6. Reaction $X e(50 \mathrm{~A} \mathrm{MeV})+S n$. Movement of the nucleons finally emitted as IMF's in forward/backward direction in the mean field potential for collisions at impact parameter $b=3 \mathrm{fm}$. We display the fraction of these nucleons on the total density (shadow) and the gradient of the potential (arrows) projected on the $x-z$ plane

\section{THE RADIAL FLOW AT BEAM ENERGIES OF 200A MEV}

In the reaction at $200 \mathrm{~A} \mathrm{MeV}$ the velocity of the nucleons due to internal motion and the velocity of the two colliding nuclei in the center of mass frame are now significantly different. As a consequence we expect a change of the reaction mechanism.

That this is indeed the case can already be seen from the time evolution of the transverse momenta of the fragments, figure 7. The collective directed in-plane flow has the opposite sign as compared to $50 \mathrm{~A} \mathrm{MeV}$. This is as well a sign for the change of the reaction mechanism which develops between $50 \mathrm{~A} \mathrm{MeV}$ and $200 \mathrm{~A} \mathrm{MeV}$ from a deep inelastic scenario with a negative flow angle to a bounce off type reaction with a positive flow angle.

Despite of the higher beam energy the transverse acceleration of the light fragments is delayed as compared to the reaction at $50 \mathrm{~A} \mathrm{MeV}$ and sets on only at the maximum overlap of the two nuclei. Then they are accelerated and remain with a high transverse momentum after the expansion.

The large fragments have a completely different time evolution as compared to $50 \mathrm{~A} \mathrm{MeV}$. First we see a deceleration in transverse direction of the fragments. Then, when the highest density is reached this turns into an acceleration. Finally, as at lower energies, the fragments are decelerated again during the expansion of the system. Finally they have a quite low transverse 


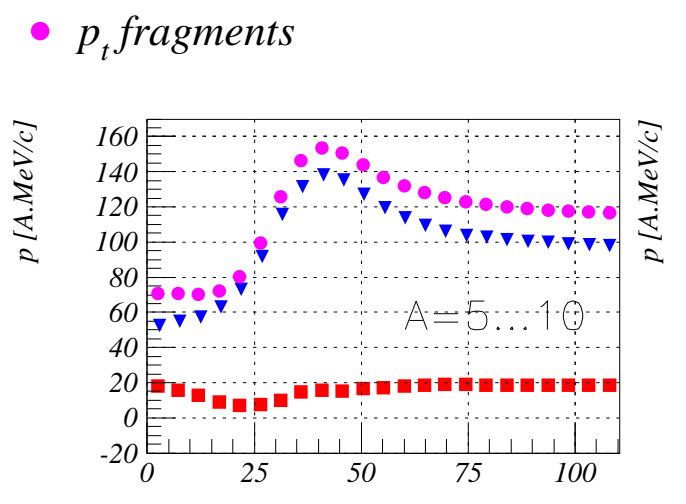

$t[\mathrm{fm} / \mathrm{c}]$

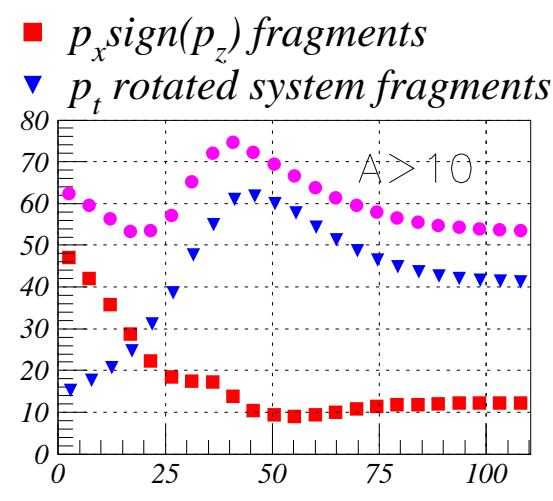

$t[\mathrm{fm} / \mathrm{c}]$

FIG. 7. Time evolution of the average transverse momentum of the IMF's with respect to the beam direction (dots) and in the rotated system (by $\theta_{\text {flow }}$ ) (triangles) for $200 \mathrm{~A} \mathrm{MeVXe}+\mathrm{Sn}, \mathrm{b}=3 \mathrm{fm}$. The squares mark the time evolution of the in-plane flow.

momentum.

To understand this behaviour it is useful to study the initial-final state correlations. Particularly in momentum space we observe such strong correlations. In figure 8 we display the initial distribution of the nucleons at $\mathrm{t}=0$ (color level) and superimpose the relative fraction of nucleons finally emitted in small (lhs) or big (middle, rhs) fragments. Here we can clearly see that the nucleons finally emitted in heavy fragments have an initial momentum which points away from the reaction partner. This initial - final - state correlation, already allusively present at $50 \mathrm{~A} \mathrm{MeV}$ increases with the mass of the fragment and the beam energy [10].

For nucleons finally emitted in small fragments the correlations are less pronounced but with preference of momenta which point into the reaction zone what favours the mixing of projectile and target nucleons in the fragments. Nucleons emitted finally in small fragments
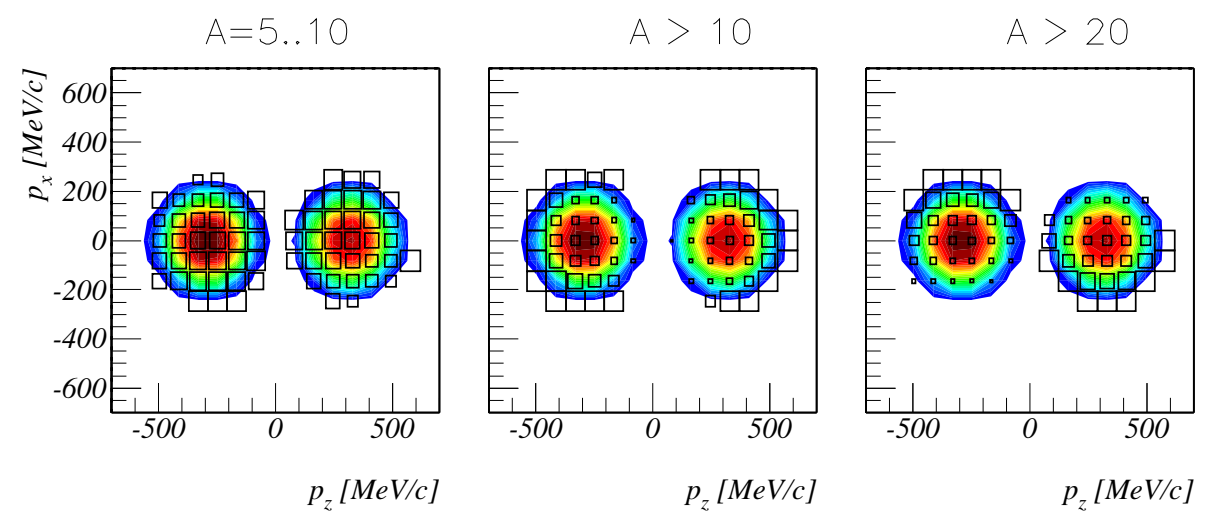

FIG. 8. Initial - final - state correlations in momentum space. We represent the positions of the nucleons at $t=0$ (color level) and superimpose as boxes the partition of nucleons finally emitted as small (lhs), big (middle) or very big (rhs) fragments.

are located in the center of the collision partners and become only accelerated when a higher density is built up in the center of the reaction. Following the density gradient they develop a radial flow. Finally they disentangle form the system. This costs energy and therefore the momentum decreases. We expect a constant acceleration of all fragment nucleons and therefore a final transverse energy which increases linearly with the fragment mass. The light fragments 
show only a small in-plane-flow which is positive in contrast to the reaction at the lower energy. In contradistinction to $50 \mathrm{~A} \mathrm{MeV}$ we observe for the light fragments a genuine acceleration in transverse direction independent of the system in which it is measured. At $200 \mathrm{~A} \mathrm{MeV}$ the density built up in the center of the reaction is sufficiently high for a noticeable acceleration of the fragments. We see that especially for the heavy fragments that the initial transverse momentum in the rotated system is quite different as compared to the beam system due to the initial correlations in momentum space, discussed above.

For a better understanding of the time evolution of the momenta of the big fragments we study as at $50 \mathrm{~A} \mathrm{MeV}$ the movement of their constituent nucleons in the nuclear potential, figure 9. Here we display the gradient of the nuclear potential and the relative fraction of nucleons emitted in fragments $A>10$.

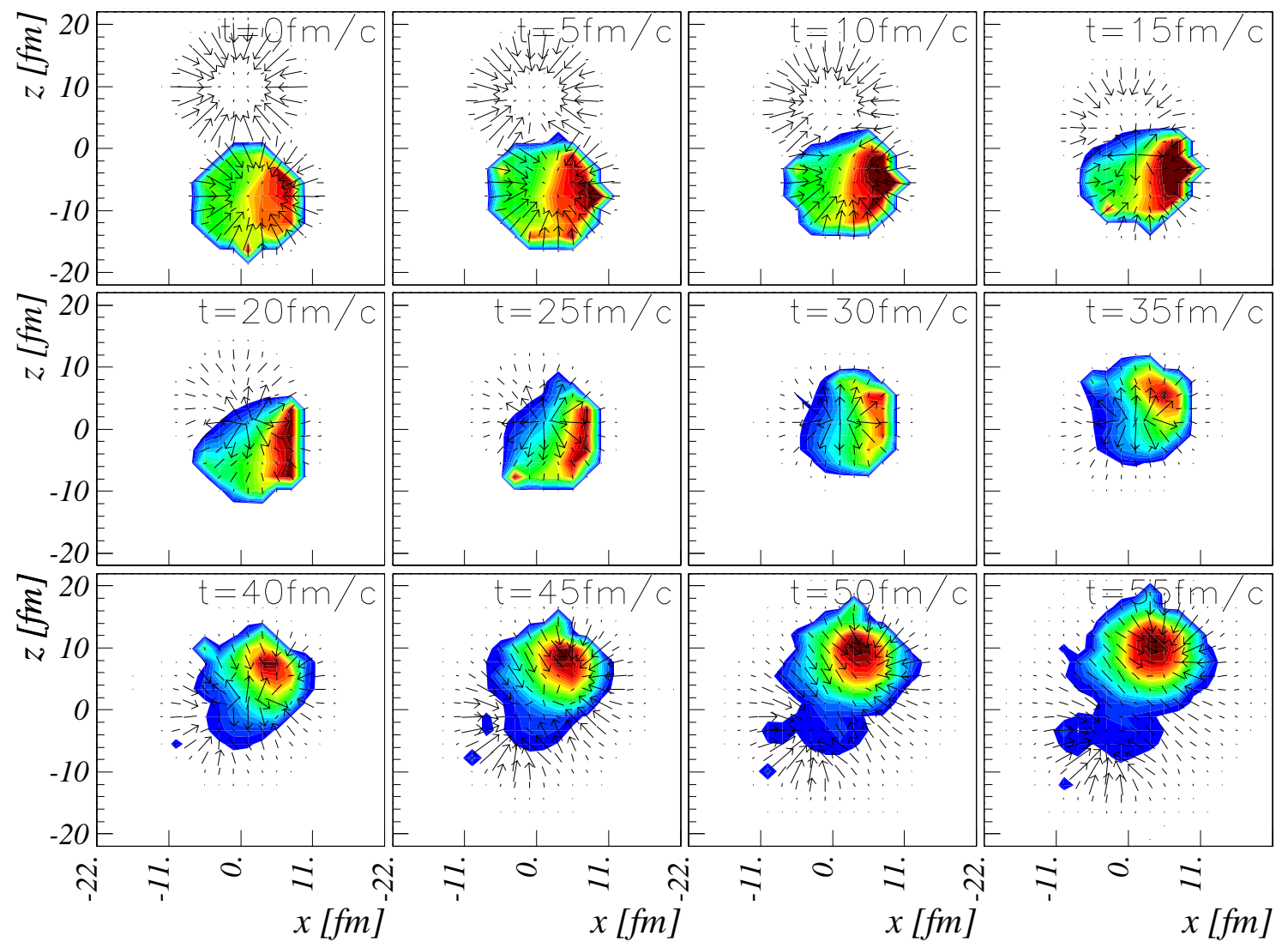

FIG. 9. Reaction Xe(200A MeV)+Sn. Movement of the nucleons finally emitted in big fragments $(A>10)$. We display the fraction of these nucleons on the total density (shadow) and the gradient of the potential (arrows) projected on the $x$-z plane

In addition to their strong initial - final - state correlations in momentum space the fragments have as well strong correlations in coordinate space. The big fragments are at $\mathrm{t}=0$ located at the surface of the collisions partners and have therefore an average density well below the normal nuclear matter density. At the beginning of the reaction they are accelerated towards the center of the reaction because the density is higher there. This lowers the transverse momentum. When the high density at the center of the reaction is built up, the acceleration changes sign. Thus we can already at $200 \mathrm{~A} \mathrm{MeV}$ and $3 \mathrm{fm}$ impact parameter distinguish between participants and spectators. 
Now we can draw up two competing processes of fragment formation at $200 \mathrm{~A} \mathrm{MeV}$ :

1. Large fragments are formed from spectator matter. The entrained nucleons do not pass the region of high density and therefore the acceleration in transverse direction is moderate. For the entrained nucleons we observe as well strong initial - final - state correlations in momentum space. In other words, the more the nucleons avoid the high density zone the larger is the change that they find themselves finally in big fragments. At $200 \mathrm{~A} \mathrm{MeV}$ the probability that nucleons passes the high density zone without having a collision is well reduced with respect to $50 \mathrm{~A} \mathrm{MeV}$. Therefore any larger correlated part of nuclear matter which passes the high density becomes destroyed by collisions and cannot form finally a fragment anymore.

2. Small correlated parts of nuclear matter can still survive the passage through the high density zone. That will be different at still higher energies. These nucleons form small fragments mixing projectile and target via collisions. The nucleons finally emitted in this fragments are close to the high density zone and accelerated due to the internal pressure. Their transverse energy is high and expected to be proportional to their mass (radial flow).
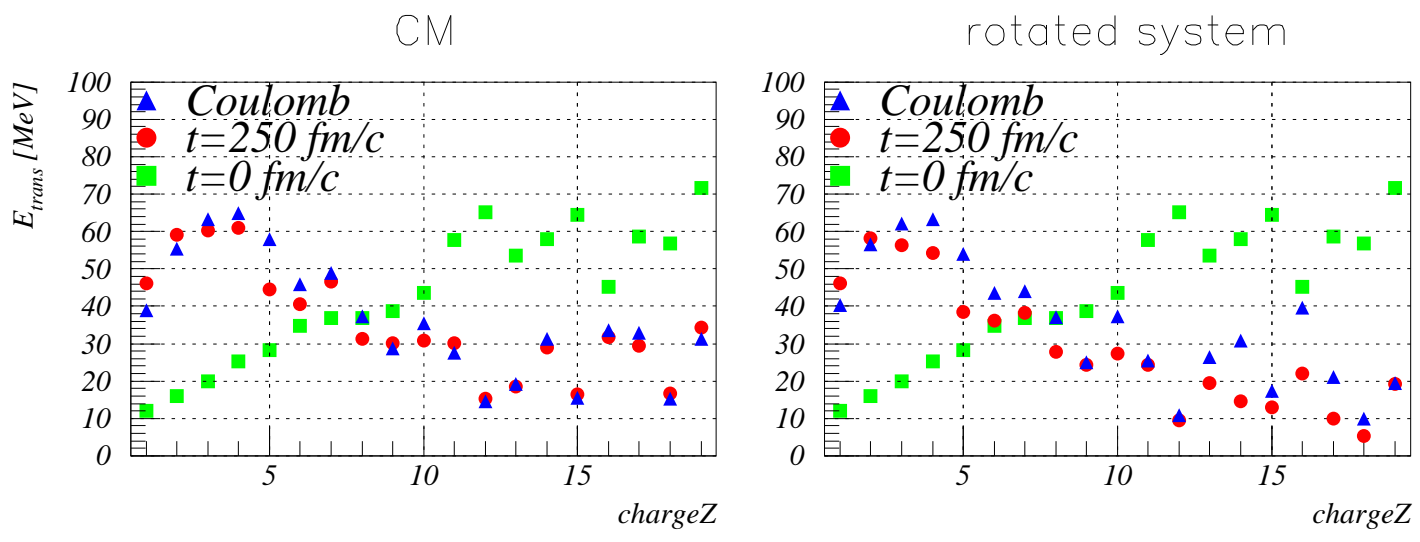

FIG. 10. Average transverse energy of fragments as a function of their charge with respect to the beam direction (left) and in the rotated system (by $\theta_{\text {flow }}$ ) (right) for $200 \mathrm{~A} \mathrm{MeVXe}+S \mathrm{Sn}, \mathrm{b}=3 \mathrm{fm}$.

These two competing processes allow to understand the behaviour of the transverse energy as a function of the charge (figure 10,lhs). At $t=0$, the beginning of the reaction, the strong initial correlations in momentum space (high transverse momentum for big fragments) cause a linear increase of the transverse energy with the charge of the fragment. For small fragments the process 2 dominates, finally the transverse energy of fragments is large and increases with increasing charge (mass) of the fragment. For big fragments the process 1 is dominant, the average transverse energy is small. In between we observe a transition phase. On the rhs of the same figure we display as in figure 3 the transverse energy of the fragments in the rotated systems which is almost identical with the left hand side. Hence the radial energies are genuine and not artifacts due to a strong in-plane flow as at $50 \mathrm{~A} \mathrm{MeV}$. 


\section{CONCLUSION}

In conclusion we have found that the kinetic energy of the fragments observed in the heavy ion reaction $\mathrm{Xe}+\mathrm{Sn}$ at $50 \mathrm{~A} \mathrm{MeV}$ reflects the initial Fermi energy of the entrained nucleons. There is no hint that the system becomes equilibrated. Rather, in agreement with experiment, even in central collisions the reaction is semi transparent. We find that the final longitudinal momentum of the fragments depends strongly on the time point when the entrained nucleons pass the center of the reaction. Nucleons which are passing early in the reaction are decelerated due to collisions with the reaction partner and due to the strong potential gradient. They are deviated into the transverse direction as their longitudinal momentum is not high enough to overcome the potential barrier formed in the reaction center in the phase of highest density. Nucleons which arrive later do not encounter a strong potential gradient anymore and pass the reaction center freely. Thus, they keep almost their initial momentum. We find that compressional effects have little influence on the final momentum of the fragments at this energy and the observed apparent radial flow is not real. The linear increase of the fragment kinetic energy with the mass for small fragments finds its natural explication in terms of the initial Fermi motion, the Coulomb barrier and a small in-plane flow.

At $200 \mathrm{~A} \mathrm{MeV}$ we found that the reaction mechanism has changed and approaches the participant-spectator model as far as heavy fragments are concerned. Due to the smaller mean free path heavy fragments cannot come anymore form the center of the reaction. Their transverse energy remains therefore moderate and the in-plane flow weak. Small fragments show a strong radial flow and have passed the center of the reaction where the forces are much stronger than at $50 \mathrm{~A} \mathrm{MeV}$.

At higher energies fragments formed from of participants become more and more rare due to the shorter mean free path and due to stronger force gradients. Hence fragment formation of spectator matter will dominate. Initial - final - state correlations in momentum space become stronger and collisions start to contribute in addition to the radial flow. How these different process influence the final radial flow and its dependence on the nuclear equation of state is presently under investigation [7].

[1] P. Braun-Munzinger et al, Phys. Lett. B344 (1995) 43 and Phys. Lett. 365 (1996) 1

[2] N. Marie, PhD Thesis, Université de Caen, France

[3] W. Reisdorf, Nucl. Phys. A612 (1997) 493, G. Poggi et al., Nucl Phys A586 (1995) 755, B. Hong et al. nucl-ex/9707001

[4] N. Herrmann, Nucl. Phys. A610 (1996) 49c,

[5] R. Nebauer at al., nucl-th/9810008

[6] J. Aichelin, Phys. Rep. 202 (1991) 233.

[7] C. Hartnack et al., to be published

[8] R. Puri et al., Phys. Rev. C54 (1996) R28

[9] A.S. Goldhaber Phys. Lett. B53 (1974) 306

[10] P.B. Gossiaux and J. Aichelin, Phys. Rev. C56 (1997) 2109 